\title{
Doxazosin Suppresses the Morning Increase in Blood Pressure and Sympathetic Nervous Activity in Patients with Essential Hypertension
}

\author{
Yoshiyuki Kawano, Osamu Tochikubo**, Yasujiro Watanabe, Eiji Miyajima**, and Masao Ishii*
}

\begin{abstract}
To investigate the effects of doxazosin on blood pressure and sympathetic nervous activity, we analyzed the circadian variation of blood pressure and the power spectrum of $R-R$ intervals using an ambulatory multibiomedical monitoring system (TM2425) in 10 untreated outpatients with essential hypertension. After a 2-wk placebo period (P-period), we administered 1 to $4 \mathrm{mg}$ of doxazosin mesilate to the patients for 2 to 6 wk (T-period). We measured systolic and diastolic blood pressure (SBP, DBP), heart rate, R$R$ intervals, posture, and activity with the use of TM2425. Power spectral analysis of $R-R$ intervals was used to calculate the ratio of low to high frequency components (LF/HF). The values were compared between the P-period and T-period. Although daytime blood pressure significantly decreased during the Tperiod (SBP, 148.1 \pm 5.9 vs. $130.3 \pm 4.4 \mathrm{mmHg}$; DBP, 92.3 \pm 3.2 vs. 83.6 $\pm 2.6 \mathrm{mmHg}, p<0.01$ ), nighttime DBP did not. The LF/HF of $R-R$ intervals in the daytime $(5.8 \pm 2.0$ vs. $4.9 \pm 1.2, p<0.01)$ and the morning rise in blood pressure also decreased significantly (SBP, 17.5 \pm 9.4 vs. 12.1 16.5 mmHg; DBP, $12.5 \pm 6.5$ vs. $8.3 \pm 5.3 \mathrm{mmHg}, p<0.05)$. We conclude that doxazosin may suppress the morning rise in blood pressure in association with a decrease in sympathetic nervous activity. (Hypertens Res 1997; 20: 149-156)
\end{abstract}

Key Words: doxazosin, essential hypertension, morning increase in blood pressure, sympathetic nervous activity

Prominent circadian variation in the frequency of cardiovascular and cerebrovascular accidents has been reported (1). Many studies have shown that the incidence of sudden cardiac death, nonfatal myocardial infarction, and episodes of myocardial ischemia is low during the nighttime and very high in the morning from 6:00 to noon (2). Cerebrovascular accidents due to thrombosis or embolism also occur more frequently during the morning (3). The hourly frequency of ventricular tachycardia in patients with acute myocardial infarction has a significant circadian variation with the highest occurrence during awake hours, similar to the rhythms described for acute myocardial infarction and sudden cardiac death (4).

Some studies have suggested a relationship between sympathetic nervous activity and cardiovascular complications. A decrease in ischemic threshold has been demonstrated in conjunction with an increase in peripheral vascular resistance in the morning (5), suggesting that increased sympathetic nerve activity may be associated with vasoconstriction. Furthermore, a study in patients with transient myocardial ischemia showed that the ratio of low to high frequency power was increased during ischemia, as demonstrated by a power spectral analysis of R-R intervals (6).

Considering these previous studies, we postulated that increased sympathetic nervous activity in the morning might contribute to a rise in blood pressure and thereby play an important role in the onset of cardiovascular accidents and, consequently, that $\alpha_{1}$ blockers may decrease blood pressure and reduce the occurrence of cardiovascular accidents in the morning. To investigate the effects of doxazosin on blood pressure and sympathetic nervous activity, we analyzed the circadian variation of blood pressure and the power spectrum of $R-R$ intervals with an ambulatory multibiomedical monitoring system.

\section{Methods}

Patients

The subjects were 10 untreated outpatients ( 5 men and 5 women, age $56 \pm 11$ [mean \pm SD] years old) with essential hypertension. The diagnosis was

From the Department of Cardiology, Cerebrovascular Center, Nanasawa Rehabilitation Hospital, Atsugi, Japan, * Second Department of Internal Medicine, School of Medicine, Yokohama City University, Yokohama, Japan, and ** Second Department of Internal Medicine, School of Medicine, Yokohama City University, Urafune Hospital, Yokohama, Japan.

Address for Reprints: Yoshiyuki Kawano, M.D., Department of Cardiology, Cerebrovascular Center, Nanasawa Rehabilitation Hospital, 1304 Nanasawa, Atsugi, Kanagawa 243-01, Japan.

Received November 25, 1996; accepted in revised form July 1, 1997. 
Table 1. Patients' Profiles, Blood Pressure, Laboratory Data, and Dose of Doxazosin

\begin{tabular}{ccccc}
\hline Age & Sex $^{1}$ & Blood Pressure $^{2}$ & Laboratory Data & Dose $^{3}$ \\
\hline 55 & $\mathrm{M}$ & $168 / 96$ & $\mathrm{CTR}=54 \%$ & $2 \mathrm{mg}$ \\
44 & $\mathrm{~F}$ & $164 / 108$ & & $4 \mathrm{mg}$ \\
53 & $\mathrm{~F}$ & $156 / 102$ & & $4 \mathrm{mg}$ \\
63 & $\mathrm{~m}$ & $154 / 98$ & & $4 \mathrm{mg}$ \\
76 & $\mathrm{M}$ & $182 / 100$ & $\mathrm{Cr}=1.8 \mathrm{mg} / \mathrm{dl}$ & $4 \mathrm{mg}$ \\
60 & $\mathrm{~F}$ & $174 / 106$ & $\mathrm{CTR}=55 \%$ & $2 \mathrm{mg}$ \\
40 & $\mathrm{~F}$ & $154 / 96$ & & $2 \mathrm{mg}$ \\
43 & $\mathrm{M}$ & $148 / 98$ & $\mathrm{CTR}=52 \%$ & $4 \mathrm{mg}$ \\
60 & $\mathrm{~F}$ & $148 / 104$ & & $4 \mathrm{mg}$ \\
69 & $\mathrm{M}$ & $168 / 104$ & & \\
\hline
\end{tabular}

${ }^{1} \mathrm{M}=$ Male, $\mathrm{F}=$ Female. ${ }^{2}$ Casual blood pressure $(\mathrm{mmHg})$ at entry to this study. ${ }^{3}$ Dose of doxazosin at the end of this study.
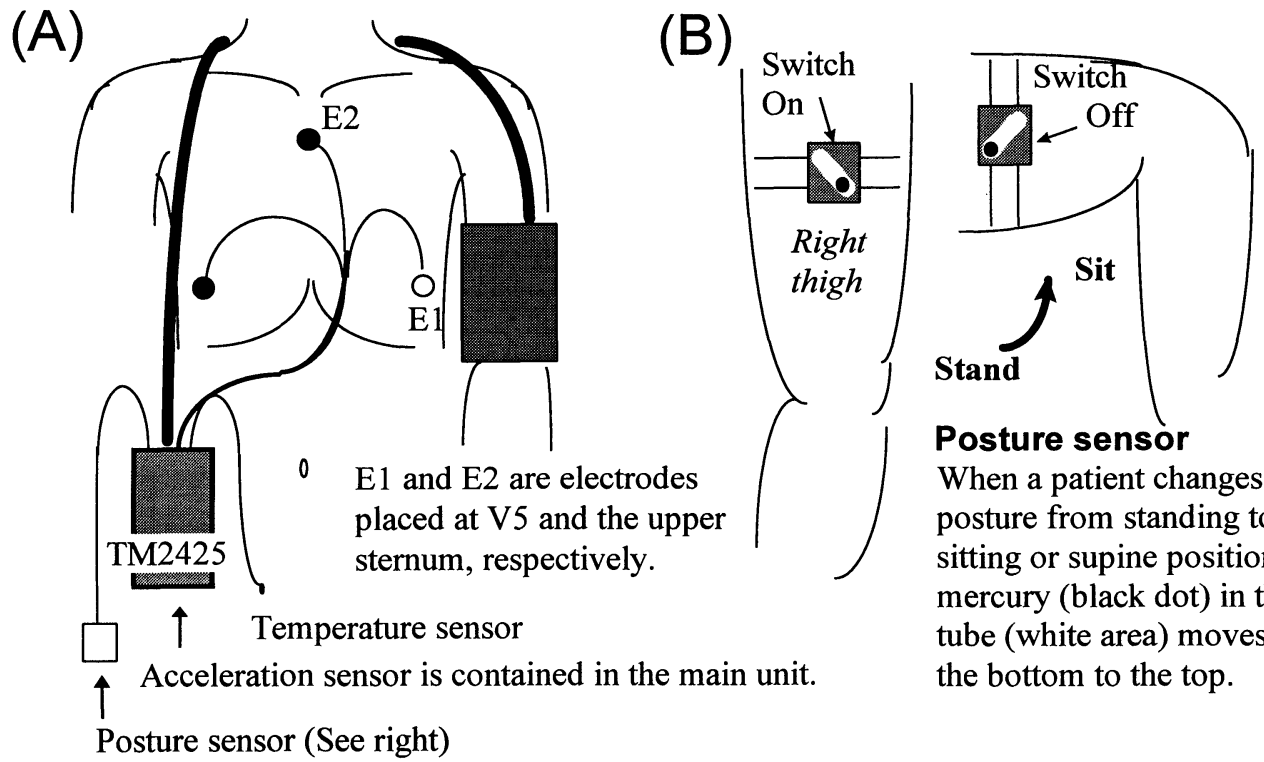

When a patient changes posture from standing to sitting or supine position, mercury (black dot) in the tube (white area) moves from the bottom to the top.

Fig. 1. Overview of the multibiomedical recorder (TM2425). The left figure (A) shows an overview of TM2425 attached to a patient. The right figure (B) illustrates how the posture sensor works.

based on blood pressure measured by conventional methods during visits to the outpatient clinic. Each patient underwent routine diagnostic procedures to exclude possible causes of secondary hypertension. Target organ damage was assessed by electrocardiogram, chest X-ray film, blood and urinary tests, and fundoscopic examination. Furthermore, patients with severe hypertension were excluded. One patient had mild renal dysfunction (creatinine $=1.8$ $\mathrm{mg} / \mathrm{dl}$ ) and three had slight cardiomegaly (Table 1).

All patients gave their informed consent to participate in the study.

\section{Measurements}

TM2425 (A\&D Co., Ltd., Tokyo, Japan) is a portable multibiomedical recorder (7) that has a cuff to measure ambulatory blood pressure, three electrodes to record the electrocardiogram, a posture sensor, an acceleration sensor, and a temperature sensor (Fig. 1)

Using this monitoring system, we recorded the systolic and diastolic blood pressure (SBP, DBP), measured by both the Korotkoff and the oscillometric methods, heart rate (HR), and R-R intervals of 512 consecutive points every $30 \mathrm{~min}$ in the nighttime and every $15 \mathrm{~min}$ in the daytime for $25 \mathrm{~h}$. Basically, we used the Korotkoff sound method to measure the blood pressure, because this method is based on the standard measurement of blood pressure with a sphygmomanometer by auscultation of Korotkoff's sounds. Blood pressure measurement with the TM2425 satisfies the accuracy levels recommended by the Association for the Advancement of Medical Instrumentation (8).

The patients' posture, defined as supine-sitting position or standing position, physical activity, measured with an acceleration sensor, and environmental temperature, measured with a thermometer placed 


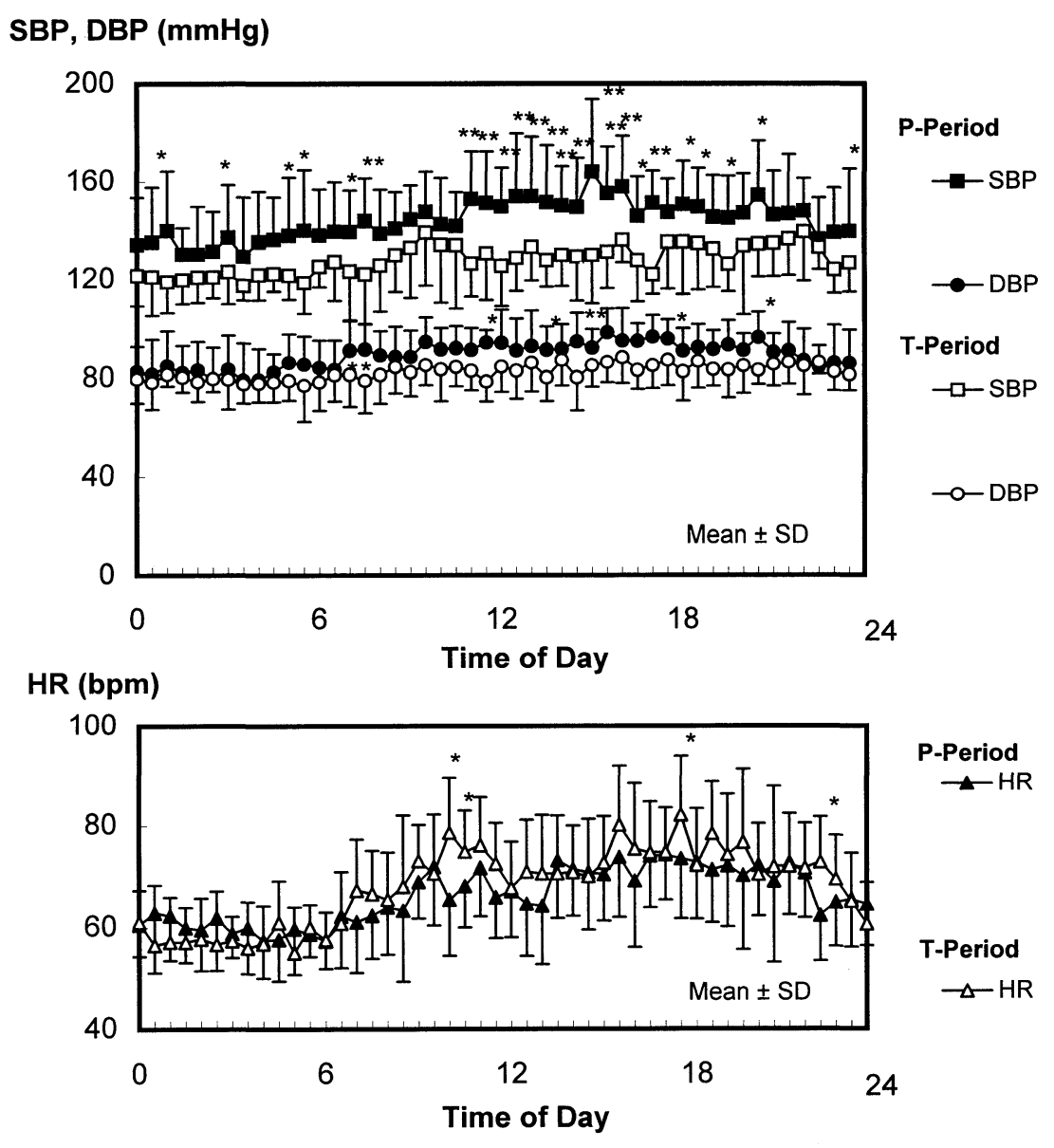

Fig. 2. Circadian pattern of blood pressure and heart rate. Mean systolic blood pressure (SBT), diastolic blood pressure $(D B P)$, and heart rate $(H R)$ were calculated every 30 min for 10 patients. Comparisons were made between the placebo period (P-period) and treatment period (T-period). ${ }^{*} \mathrm{p}<0.05, * * \mathrm{p}<0.01$.

outside the patients' clothes, were recorded every minute (Fig. 1).

We measured the blood pressure and heart rate for 15-min intervals in the daytime (from 6:00 to 21:00) and 30-min intervals in the nighttime (from 21:00 to $6: 00$ of the next morning). To evaluate the circadian variation of blood pressure and heart rate, we calculated the mean data of all patients for every 30 -min interval. We defined the blood pressure measured within $15 \mathrm{~min}$ before a patient got up as

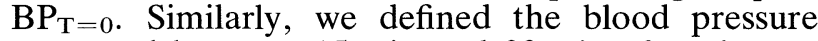
measured between $15 \mathrm{~min}$ and $30 \mathrm{~min}$ after the patient got up as $\mathrm{BP}_{\mathrm{T}=15}$. Thus, we synchronized the time to evaluate the morning rise in blood pressure, which was calculated as $\mathrm{BP}_{\mathrm{T}=15}-\mathrm{BP}_{\mathrm{T}=0}$.

\section{Protocol}

After the diagnostic procedures were completed, placebo was given to the patients for $2 \mathrm{wk}(\mathrm{P}$ period). At the end of the P-period, blood pressure was measured for $25 \mathrm{~h}$ with TM2425. Thereafter, the patients whose blood pressure remained above $140 / 90 \mathrm{mmHg}$ were given $1 \mathrm{mg}$ doxazosin mesilate (Cardenalin ${ }^{\circledR}$; Pfizer Inc., New York, USA). During the treatment period (T-period), doxazosin was given to the patients once in the morning. The effect of treatment was judged on the basis of the blood pressure measured $2 \mathrm{wk}$ after the start of treatment. If the blood pressure was below 140/90 $\mathrm{mmHg}$, or if the systolic blood pressure was 20 $\mathrm{mmHg}$ lower or the diastolic blood pressure was 10 $\mathrm{mmHg}$ lower than during the P-period, the treatment was evaluated to be effective. We then measured the blood pressure for $25 \mathrm{~h}$ with TM2425, and we ended the study. If the treatment was ineffective, we increased the dose to $2 \mathrm{mg} / \mathrm{d}$ and measured blood pressure $2 \mathrm{wk}$ later. If the treatment was still ineffective, we further increased the dose to a maximum of $4 \mathrm{mg} / \mathrm{d}$ and repeated the procedures.

Because patients may be nervous when unaccustomed to ambulatory blood pressure monitoring (ABPM) and their blood pressure may be affected, we attached the ABPM device to the patients for 25 $\mathrm{h}$ and discarded data from the first hour. We instructed the patients to get up at 6:00 and go to bed at 21:00 and to act as usual during the daytime. To study the morning rise in blood pressure, however, we synchronized the precise get-up time based on their record of daily activity before we analyzed the data of all patients. 
Table 2. Comparisons of Data between Placebo and Treatment Periods

\begin{tabular}{|c|c|c|c|}
\hline & P-period & T-period & $p$-value \\
\hline \multicolumn{4}{|l|}{ Daytime } \\
\hline SBP (mmHg) & $148.1 \pm 5.9$ & $130.3 \pm 4.4$ & $<0.01$ \\
\hline DBP (mmHg) & $92.3 \pm 3.2$ & $83.6 \pm 2.6$ & $<0.01$ \\
\hline HR (bpm) & $68.6 \pm 4.4$ & $71.8 \pm 5.1$ & n.s. \\
\hline Pos & $6.5 \pm 2.3$ & $11.6 \pm 2.7$ & $<0.01$ \\
\hline Act $(G)$ & $0.27 \pm 0.09$ & $0.49 \pm 0.19$ & $<0.01$ \\
\hline $\mathrm{LF} / \mathrm{HF}$ & $5.8 \pm 2.0$ & $4.9 \pm 1.2$ & $<0.01$ \\
\hline \multicolumn{4}{|l|}{ Nighttime } \\
\hline SBP (mmHg) & $137.0 \pm 5.1$ & $128.0 \pm 6.2$ & $<0.05$ \\
\hline DBP (mmHg) & $84.0 \pm 3.1$ & $82.6 \pm 2.9$ & n.s. \\
\hline HR (bpm) & $61.5 \pm 3.3$ & $60.6 \pm 5.5$ & n.s. \\
\hline Pos & $5.9 \pm 1.9$ & $5.5 \pm 2.6$ & n.s. \\
\hline Act $(G)$ & $0.12 \pm 0.06$ & $0.15 \pm 0.14$ & n.s. \\
\hline $\mathrm{LF} / \mathrm{HF}$ & $5.7 \pm 1.8$ & $6.5 \pm 1.6$ & $<0.05$ \\
\hline \multicolumn{4}{|l|}{ 24-h } \\
\hline SBP $(\mathrm{mmHg})$ & $144.1 \pm 7.7$ & $128.1 \pm 5.9$ & $<0.01$ \\
\hline DBP (mmHg) & $89.4 \pm 5.1$ & $82.6 \pm 3.1$ & $<0.05$ \\
\hline $\mathrm{HR}(\mathrm{bpm})$ & $66.1 \pm 5.3$ & $67.9 \pm 7.5$ & n.s. \\
\hline Pos & $6.3 \pm 2.2$ & $9.8 \pm 3.6$ & $<0.01$ \\
\hline Act $(\mathrm{G})$ & $0.21 \pm 0.11$ & $0.37 \pm 0.24$ & $<0.05$ \\
\hline $\mathrm{LF} / \mathrm{HF}$ & $5.7 \pm 1.9$ & $6.0 \pm 1.7$ & n.s. \\
\hline
\end{tabular}

Systolic blood pressure (SBP), diastolic blood pressure (DBP), heart rate (HR), posture (Pos), activity (Act) and the ratio of low and high frequency components of $\mathrm{R}-\mathrm{R}$ intervals $(\mathrm{LF} / \mathrm{HF})$ in hypertensive patients during the placebo period (P-period) and the treatment period (T-period). Data shown are means $\pm S D$. As for posture, sitting and standing positions were recorded as 0 and 1 , respectively. Activity was measured as acceleration.

This protocol was approved by the ethics committee of Yokohama City University, School of Medicine.

\section{Power Spectral Analysis of $R-R$ Intervals}

R-R intervals of 512 points recorded by TM 2425 were resampled at $500-\mathrm{ms}$ intervals. These data were analyzed by means of Fast Fourier Transform. We divided the power spectrum obtained as described above into a low frequency spectrum (LF: 0.04 to $0.15 \mathrm{~Hz}$ ) and a high frequency spectrum (HF: 0.15 to $0.40 \mathrm{~Hz}$ ). Furthermore, we calculated the ratio (LF/HF) between these two values (9).

Postural Changes in Blood Pressure and Heart Rate We classified the recorded SBP according to whether the patients were in the supine or sitting position (SBPsit) or in the standing position (SBPstd). The difference between these two values was defined as the postural change in SBP $(\triangle \mathrm{SBP}=$ SBPstd - SBPsit). Similarly, we calculated the postural changes in DBP and HR ( $\triangle \mathrm{DBP}, \triangle \mathrm{HR})$.

\section{Statistical Analysis}

We compared the data obtained during the placebo and treatment periods for each patient. Student's $t$ test was used for statistical analysis, and differences with a $p$ value of less than 0.05 were considered as statistically significant.

\section{Results}

Circadian Variation in Blood Pressure and Heart Rate

After doxazosin was given to the patients, daytime (6:00-21:00), but not nighttime (21:00-6:00), blood pressure decreased significantly (Fig. 2). The mean \pm SD of daytime, nighttime, and $24-\mathrm{h}$ monitoring values for each variable during the P-period and $\mathrm{T}$ period are shown in Table 2. During the daytime, significant decreases were observed during the $\mathrm{T}$ period for all variables except heart rate. As for nighttime values, only SBP and LF/HF showed a significant decrease during the $\mathrm{P}$-period.

Circadian Variation in $L F / H F$ of $R-R$ Intervals The LF of R-R intervals was higher in the P-period than in the T-period in the morning $\left(359 \pm 165 \mathrm{~ms}^{2}\right.$ vs. $\left.284 \pm 120 \mathrm{~ms}^{2}, p<0.05\right)$. On the other hand, HF was lower in the P-period than in the T-period in the morning $\left(98 \pm 51 \mathrm{~ms}^{2}\right.$ vs. $154 \pm 69 \mathrm{~ms}^{2}, p<$ $0.05)$. In the P-period, the $L F / H F$ ratio of $R-R$ intervals rose early in the morning, although it was low in the afternoon and at night. In contrast, in the T-period, the LF/HF ratio of R-R intervals decreased in the morning (Fig. 3). The daytime $\mathrm{LF} / \mathrm{HF}$ ratio of R-R intervals decreased significantly during the T-period from $5.8 \pm 2.0$ to $4.9 \pm 1.2(p<$ 0.01 , Table 1). 

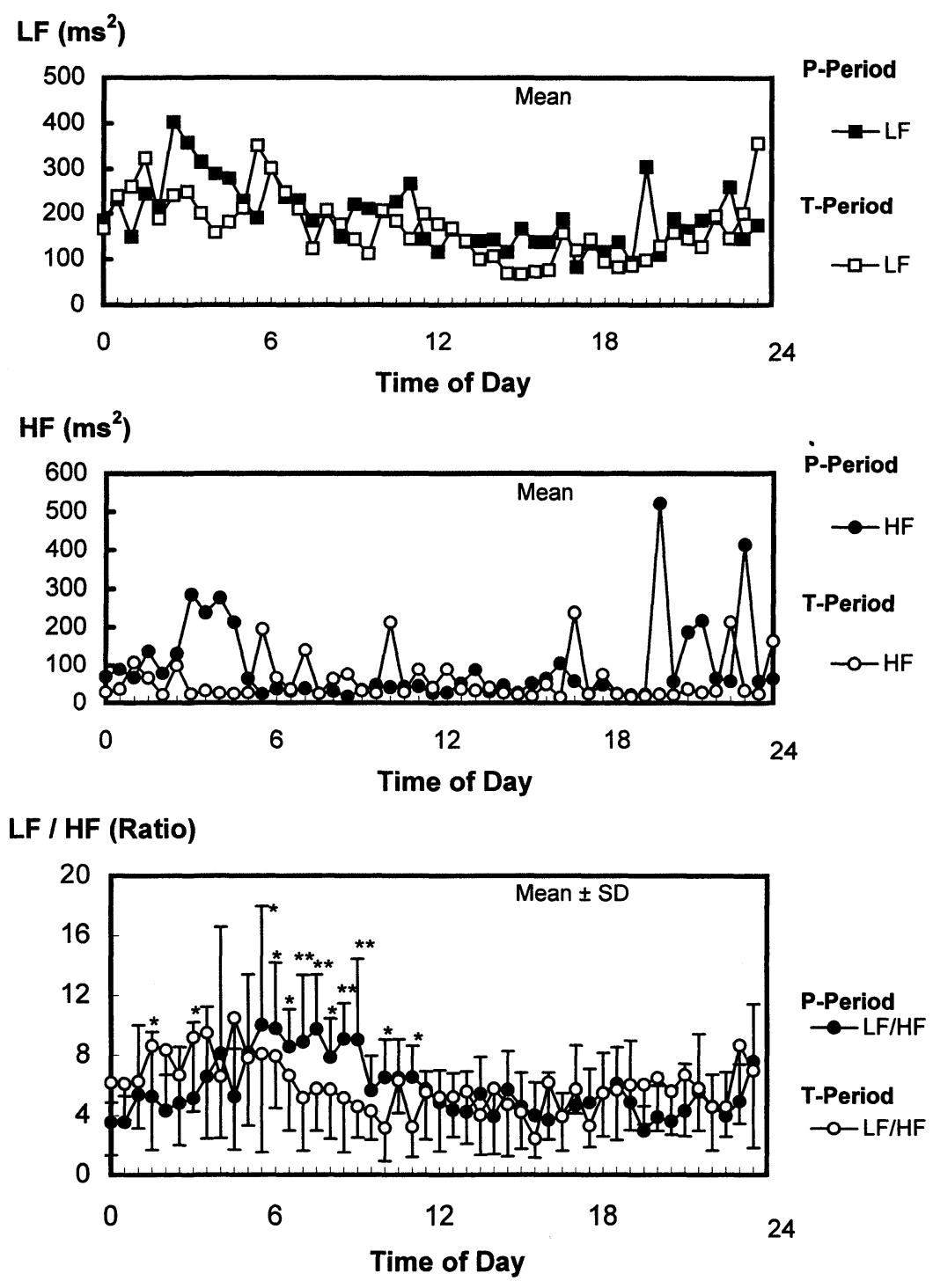

Fig. 3. Circadian pattern of power spectral components of $R-R$ intervals. Mean low frequency component (LF: 0.04-0.15 $H z)$ and high frequency component $(H F: 0.15-0.40 \mathrm{~Hz})$ of $R$ - $R$ intervals and their ratio $(L F / H F)$ were calculated every 30 min. Comparisons were made between the placebo period (P-period) and treatment period (T-period). ${ }^{*} \mathrm{p}<0.05,{ }^{* *} \mathrm{p}<$ 0.01 .

\section{Morning Rise in Blood Pressure}

The morning rise in blood pressure was higher in the P-period than in the T-period $(17.5 \pm 9.4 \mathrm{mmHg}$ vs. $12.1 \pm 6.5 \mathrm{mmHg}, p<0.05)$. Similarly, the rise in DBP was higher in the P-period than in the Tperiod $(12.5 \pm 6.5 \mathrm{mmHg}$ vs. $8.3 \pm 5.3 \mathrm{mmHg}, p<$ $0.05)$. However, the rise in HR did not significantly differ between the P-period and the T-period (Fig. 4).

\section{Variation in Blood Pressure According to Postural Changes}

Postural changes during the daytime did not affect blood pressure or $\mathrm{HR}$ in a significantly different manner during the P-period and the T-period (Fig. $5)$.

\section{Discussion}

The frequency of ischemic cardiovascular accidents peaks in the morning, with $39 \%$ of events occurring between 6:00 a.m. and noon. Similarly, $46 \%$ of ischemic changes on ECG, with or without symptoms, occur during the morning hours (10). Some reports emphasized the relationship between the morning rise in blood pressure and the increase in cardiovascular events in the morning (11).

Our data on the circadian variation of blood pressure showed that it started to rise early in the morning, maintained a high value during daytime, and fell at night. All of our subjects were outpatients, and they arose at different times in the morning. Therefore, when blood pressure values of 
SBP $(\mathbf{m m H g})$

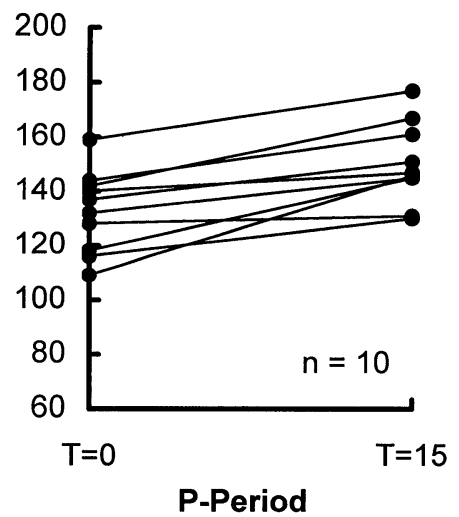

$\mathrm{DBP}(\mathrm{mmHg})$

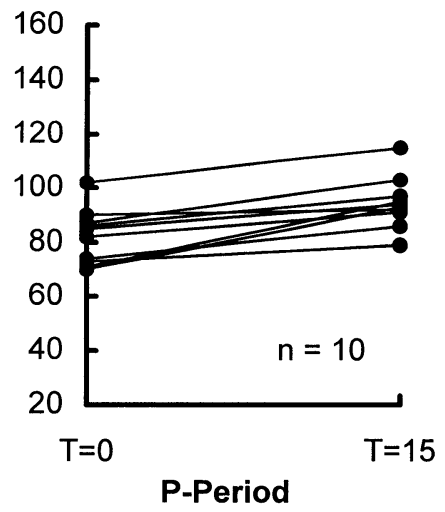

\section{HR (bpm)}

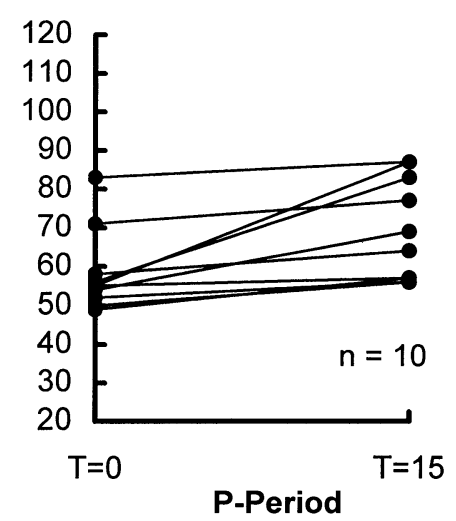

SBP $(\mathrm{mmHg})$

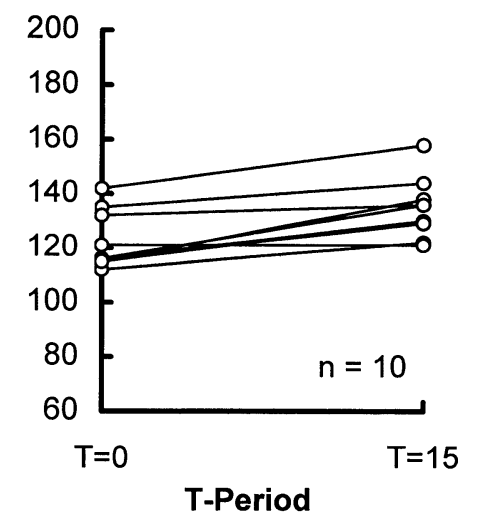

DBP ( $\mathrm{mmHg})$

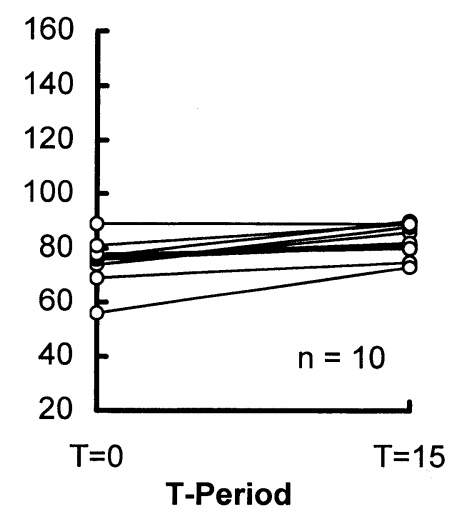

HR (bpm)

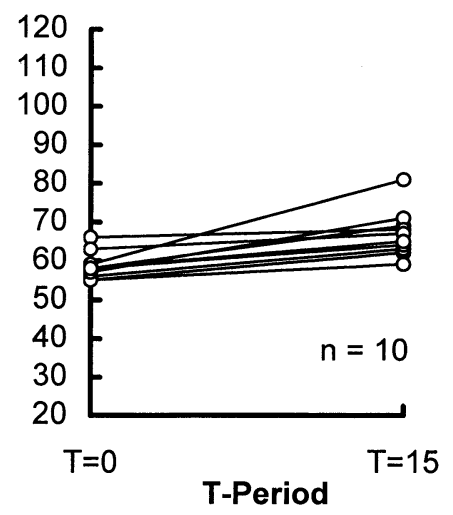

Fig. 4. The morning rise in blood pressure and heart rate. For each patient, the differences in systolic blood pressure $(S B P)$, diastolic blood pressure $(D B P)$, and heart rate $(H R)$ between when blood pressure was measured within 15 min before awakening $(\mathrm{T}=0)$ and when blood pressure was measured between 15 and 30 min after awakening $(\mathrm{T}=15)$ were calculated. The differences were compared between the placebo period (P-period) and treatment period (T-period).

the whole study population were averaged, the rise in blood pressure in the morning was apparently more gradual than it is in a synchronized study. On an individual basis, however, we observed that blood pressure rose steeply in the morning. Our data showed that the rise in blood pressure between 15 and $30 \mathrm{~min}$ after waking up in the morning decreased after treatment with doxazosin. All patients received doxazosin treatment after the placebo period. The possibility that this method may affect the result of comparisons between the placebo and treatment period should be considered as a study limitation. We instructed the patients to arise at 6:00 and to go to bed at 21:00. Because this schedule may have differed from their usual activity pattern, such instructions may have affected the results.

The morning rise in blood pressure has been re- 

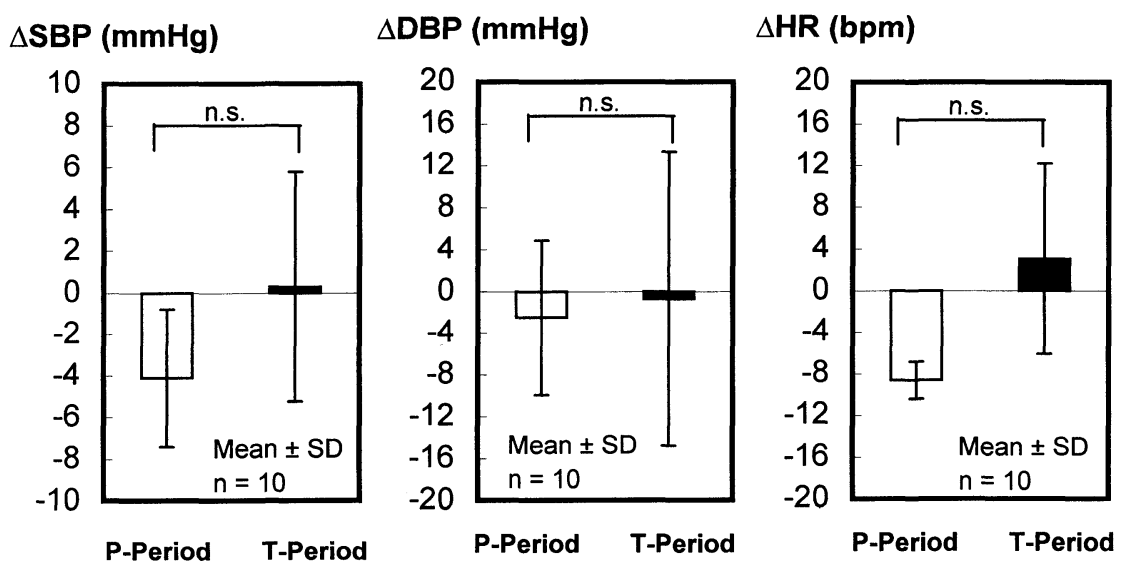

Fig. 5. Postural changes in blood pressure and heart rate. The difference in systolic blood pressure between standing position (SBPstd) and sitting or supine position (SBPsit) was calculated ( $\triangle S B P=S B P$ std $-S B P$ sit). Similarly, the differences in diastolic blood pressure $(\triangle D B P)$ and heart rate $(\triangle H R)$ between standing position and sitting or supine position were calculated. For each difference, comparison was made between the placebo period (P-period) and treatment period (T-period).

ported to be an important risk factor for cardiovascular accidents (12). Sudden changes in coronary intraluminal pressure or arterial tone during heart contractions may contribute to plaque disruption (13), which is implicated in the onset of cardiovascular and cerebrovascular accidents (14). Therefore, medical treatment to suppress the morning rise in blood pressure may help prevent the occurrence of cerebrovascular and cardiovascular accidents. Measurement of the morning rise in blood pressure has also been reported to be useful in assessing risk (11). We measured the morning rise in blood pressure during a short period as compared with previous studies, but obtained similar results. The maximum rise in systolic blood pressure 3 $\mathrm{h}$ after getting up in the $\mathrm{P}$-period and $\mathrm{T}$-period was $20.1 \pm 11.5$ and $10.9 \pm 6.4 \mathrm{mmHg}$, respectively $(p<$ $0.05)$. However, the rise in blood pressure $3 \mathrm{~h}$ before getting up was similar in the P-period and Tperiod $(14.4 \pm 9.1 v s .11 .8 \pm 8.9 \mathrm{mmHg}$, n.s.). Either the limited number of subjects or the presence of orthostatic hypotension associated with doxazosin treatment may have affected our results.

For each subject, we confirmed that the microphone was not dislodged at the end of the study and that similar results were obtained when BP was measured by the oscillometric method.

Some investigators have implicated $\alpha$-sympathetic nervous activity in the morning increase in cardiovascular accidents (15). Although $\alpha_{1}$-blockers may increase intracardiac $\beta$-adrenergic activity, many previous studies have found that doxazosin treatment does not significantly change heart rate (16). In our study, there was also no significant difference in heart rate between the P-period and Tperiod. The effects of $\alpha_{1}$-blockers on the central nervous system, cardiac muscle, or baroreflex activity may be responsible for no change in heart rate (17). Furthermore, an increase in total peripheral vascular resistance in conjunction with ST-segment depression on the electrocardiogram, and a decrease in baroreceptor sensitivity in the morning have been reported $(5,18)$. Our data showed that the $\mathrm{LF} / \mathrm{HF}$ ratio of $\mathrm{R}-\mathrm{R}$ intervals in the morning increased in the P-period and decreased after treatment with doxazosin.

In this study, we did not measure direct beat-tobeat blood pressure and did not analyze the power spectrum of blood pressures. Instead, we used the power spectrum of R-R intervals to evaluate sympathetic nervous activity on the basis of a previous study suggesting that the increase in LF component of R-R variability is induced by the Meyer wave of blood pressure variability through the baroreflex (19). However, the same report also suggested that LF component of R-R variability may not be a consistent marker of sympathetic vasomotor regulation. A previous study also reported that angina caused by coronary arterial spasm can be induced by stimulation of $\alpha$-adrenergic receptors (20). Therefore, treatment of hypertension with $\alpha_{1}$-blockers may prevent the occurrence of cardiovascular accidents in the morning.

Furthermore, increased hematocrit and enhanced coagulability of the blood in the morning have been reported as risk factors for thromboembolic cardiovascular accidents (21). Increased sympathetic nervous activity may be related to an increase in platelet aggregability in the morning, which in turn may contribute to the risk of cardiovascular accidents (22).

In our study, distinction between sitting position and supine position was impossible because the TM2425 has only one mercury switch. In general, however, orthostatic hypotension is regarded as a fall in blood pressure between the sitting position and standing position or between the supine position and standing position. Therefore, we did not address the differences in blood pressure between the sitting and supine position; such differences are 
now under investigation.

Some previous studies have reported an effect of doxazosin on the circadian variation of blood pressure $(23,24)$. They suggested that the morning rise in blood pressure may be associated with increased sympathetic nervous activity. After the administration of doxazosin twice daily, the increase in blood pressure during the early morning was suppressed in association with attenuation of $\alpha$-adrenergic activity (25). In another study, the increase in blood pressure in the morning of the next day was suppressed after a single daily dose of doxazosin (26). Sympathetic nervous activity involved in elevating blood pressure during the night may be higher in "nondippers" than in "dippers" (25). The time of peak effect may depend on the prevailing level of $\alpha$ adrenergic tone as well as on drug pharmacokinetics (27). The possibility that doxazosin attenuates sympathetic nervous activity, as estimated by $\mathrm{LF} / \mathrm{HF}$ ratio of $R-R$ intervals, warrants further investigation.

\section{References}

1. Muller JE, Ludmer PL, Willich SN, et al: Circadian variation in the frequency of sudden cardiac death. Circulation 1987; 75: 131-138.

2. Muller JE, Stone PH, Turi ZG, et al: Circadian variation in the frequency of onset of acute myocardial infarction. N Engl J Med 1985; 313: 1315-1322.

3. Agnoli A, Manfredi M, Mossuto L, Piccinelli A: Relationship between circadian rhythm and blood pressure and the pathogenesis of cerebrovascular insufficiency (in French). Rev Neurol 1975; 131: 597-606.

4. Lucente M, Rebuzzi AG, Lanza GA, et al: Circadian variation of ventricular tachycardia in acute myocardial infarction. Am J Cardiol 1988; 62: 670-674.

5. Quyyumi AA, Panza JA, Diodati JG, Lakatos E, Epstein SE: Circadian variation in ischemic threshold: a mechanism underlying the circadian variation in ischemic events. Circulation 1992; 86: 2228.

6. van Boven AJ, Brouwer J, Crijns HJGM, Haaksma J, Lie KI: Differential autonomic mechanisms underlying early morning and daytime transient myocardial ischaemia in patients with stable coronary artery disease. Br Heart J 1995; 73: 134-138

7. Tochikubo O, Ikeda A, Miyajima E, Ishii M: Effects of insufficient sleep on blood pressure monitored by a new multibiomedical recorder. Hypertension 1996; 27: 1318-1324.

8. American National Standard: Electronic or automated sphygmonanometers. Arlington, Association for the Advancement of Medical Instrumentation, 1993, pp 1-39.

9. Malliani A, Pagani M, Lombardi F, Cerutti S: Cardiovascular neural regulation explored in the frequency domain. Circulation 1991; 84: 482-492.

10. Rocco MB, Barry JJ, Campbell S, et al: Circadian variation of transient myocardial ischemia in patients with coronary artery disease. Circulation 1987; 75: 395-400.

11. Kuwajima I, Mitani K, Miyao M, Suzuki Y, Kuramoto K, Ozawa T: Cardiac implications of the morning surge in blood pressure in elderly hypertensive patients: relation to arising time. Am J Hypertens 1995; 8: 29-33.
12. Millar-Craig MW, Bishop CN, Raftery EB: Circadian variation of blood-pressure. Lancet 1978; 1: 795-797.

13. Fuster V, Badimon L, Badimon JJ, Chesebro JH: The pathogenesis of coronary artery disease and the acute coronary syndromes. N Engl J Med 1992; 326: 242-254.

14. Davies MJ, Thomas AC: Plaque fissuring: the cause of acute myocardial infarction, sudden ischemic death, and crescendo angina. Br Heart $J$ 1985; 53: 363-373.

15. Yasue H, Omote $\mathbf{S}$, Takizawa A, Nagao M, Miwa $K$, Tanaka S: Circadian variation of exercise capacity in patients with Prinzmetal's variant angina: role of exercise-induced coronary arterial spasm. Circulation 1979; 59: 938-948.

16. Tsukiyama $\mathrm{H}$, Otsuka $\mathrm{K}$, Horii $\mathrm{M}$, Takasaki I: Hemodynamic effects of tilisolol, a new $\beta$-adrenergic blocking agent with vasodilating property, and doxazosin, a new $\alpha$-adrenergic blocking agent, in essential hypertension. Jpn J Clin Pharmacol Ther 1988; 19: 511-517.

17. Tsunoda $\mathrm{K}$, Abe $\mathrm{K}$ : $\alpha$-adrenergic receptor blockade. Nihon-Rinsyo 1992; 50: 703-714.

18. Kawano Y, Tochikubo O, Minamisawa K, Miyajima $\mathrm{E}$, Ishii $\mathrm{M}$ : Circadian variation of haemodynamics in patients with essential hypertension: comparison between early morning and evening. J Hypertens 1994; 12: $1405-1412$.

19. Parati G, Saul JP, Di Rienzo M, Mancia G: Spectral analysis of blood pressure and heart rate variability in evaluating cardiovascular regulation: a critical appraisal. Hypertension 1995; 25: 1276-1286

20. Yasue H, Touyama M, Kato H, Tanaka S, Akiyama F: Prinzmetal's variant form of angina as a manifestation of alpha-adrenergic receptor-mediated coronary artery spasm: documentation by coronary arteriography. Am Heart J 1976; 91: 148-155.

21. Gagnon DR, Zhang TJ, Brand FN, Kannel WB: Hematocrit and the risk of cardiovascular disease: the Framingham study: a 34-year follow-up. Am Heart $J$ 1994; 127: 674-682.

22. Tofler GH, Brezinski D, Schafer AI, et al: Concurrent morning increase in platelet aggregability and the risk of myocardial infarction and sudden cardiac death. $N$ Engl J Med 1987; 316: 1514-1518.

23. Ebata $H$, Hojo $Y$, Ikeda $U$, Ishida $H$, Natsume $T$, Shimada K: Different effect of $\alpha_{1}$-blocker (doxazosin) on diurnal blood pressure variation in dipper and non-dipper type hypertension. Ther Res 1994; 15: 461-468.

24. Hayashi $\mathrm{H}$, Ohno $\mathbf{M}$, Ogawa $\mathrm{S}$, et al: The effect of doxazosin on circadian rhythm of blood pressure in patients with essential hypertension. Ther Res 1991; 12: 529-537.

25. Ebata $H$, Hojo $Y$, Ikeda $U$, Ishida $H$, Natsume $T$, Shimada K: Differential effects of an $\alpha_{1}$-blocker (doxazosin) on diurnal blood pressure variation in dipper and non-dipper type hypertension. Hypertens Res 1995; 18: 125-130.

26. Kuwajima I, Toyoshima T, Nakamura M, Yamashina A, Takao N: Effects of doxazosin mesilate on the morning increase of blood pressure (in Japanese). Ketsuatu 1996; 3: 388-393.

27. Pickering TG, Levenstein $M$, Walmsley $P$, the Hypertension and Lipid Trial Study Group: Night time dosing of doxazosin has peak effect on morning ambulatory blood pressure: results of the HALT study. Am J Hypertens 1994; 7: 844-847. 